\title{
Design and Implementation of Instrument NA Based on WMN
}

\author{
Fangfei Wu ${ }^{1,}$, Meihua Yu ${ }^{2}$, Jun Peng ${ }^{1, *}$, Yina Guo ${ }^{3}$ \\ ${ }^{1}$ Nanchang Institute of Technology, Nanchang 330018, China \\ ${ }^{2}$ Jiangxi water resources institute \\ ${ }^{3}$ Bangshan health-center, Longhai \\ a54703916@qq.com \\ *Corresponding author
}

Keywords: WMN, NA, AODV Routing Protocol, HiSLIP

\begin{abstract}
In order to realize network for instrumentation without network interface, current instrumentation's network was studied. It adopted AODV routing protocol in wireless Mesh network and designed multiple-interface instrumentation network agent, which could connect network and control terminal interconnection. Agent communicated with host by High Speed LAN Instrument Protocol (HiSLIP), and forwarded conveyed message of host to instrumentation through virtual instrument software architecture (VISA) interface, in order to achieve user remote control of instrument.
\end{abstract}

\section{Introduction}

With the coming of Internet plus era, networking of instrumentation has already become irresistible trend. Agilent Technologies and VXI Technology established LXI Alliance in 2004. Strictly based on IEEE 802.3, TCP/IP, Network bus, Web browser, IVI-COM Driver, Clock synchronization protocol (IEEE1588) and standard module size, networking of instrumentation come true ${ }^{[1]}$. However, early production of instrumentation did not have a network interface in current various laboratory; Even though part of sub-new equipment equipped with network interface, it still brought some inconvenience to instrumentation network which due to the vulnerable of network cabling and line.

Therefore, it is extremely urgent to design and study the network agent of instrument based on Wireless Mesh Network. WMN is Oriented IP access new wireless mobile communication technology, which is suitable for regional environmental coverage and broadband wireless access. Wireless Mesh Network is the cooperation and coordination of numerous net distributed wireless points and has its advantage of broadband high-speed and high efficiency and its prominent characteristics of dynamic self-organization, self-configuration and self-maintenance [2]. Instrumentation without network interface could connect WMN through instrument NA in order to achieve the network of instrumentation.

\section{General Framework}

Instrument NA framework based on WMN is shown as Fig. 1. It consists of Wireless Mesh Network, instrument agent, instrumentation and user control terminal. Embedded development platform was adapted to realize instrument NA function and Mesh routing function. NA and instrumentation were linked by the interface bus came with instrumentation, then achieved interconnection with user control terminal and accessed Internet by WMN, which user used virtual device soft panel for various operations on the control terminal. 


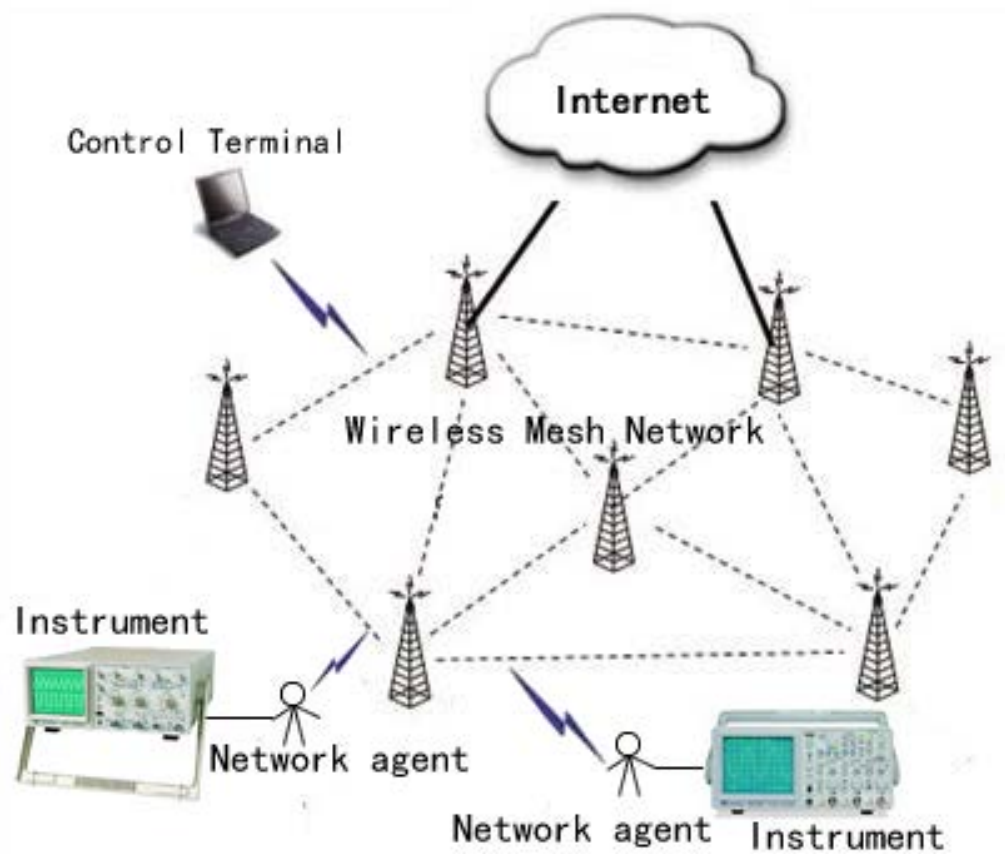

Fig. 1 Instrument NA framework based on WMN

\subsection{Wireless Mesh Network Structure Design}

Wireless Mesh Network (WMA) consisted of Mesh router and Mesh terminal, as shown in Fig. 1. Wireless Mesh Network framework consisted by Mesh router, was linked with wire network, which provided multi-hop wireless network connections ${ }^{[3]}$. Comparing with traditional wireless network, it is a wireless network architecture which had novel bandwidth.

This architecture adapted multi-level structural WMN. This structure was divided into two parts, internal part was a Mesh LAN, which interconnected by multiple Mesh routers, then linked with the nearest router. It made the entire WMN access to the Internet; external part could be desktop, intelligent terminal. Mesh router in LAN not only have traditional wireless router gateway and relay function ${ }^{[4]}$, but also has interconnected router function. Inter-node data forwarded to destination by adjacent multiple Mesh routes and obtained same wireless coverage range as lower power.

\subsection{Agent Design}

Instrumentation communicated with control terminal, at the same time, it was still interacted with instrument ${ }^{[5]}$. Asynchronous channel and synchronization channel existed between instrument and control terminal. Asynchronous channel was used for transmission of special events and special state; synchronization channel was used for transmission of common control command and measured data, which needed to guarantee the sequence of sending and receiving messages. Relevant data could be shared between asynchronous channel and synchronization channel. In order to guarantee the reliability of the link, when agent communicated with control terminal by High-speed LAN instrument protocol (HiSLIP), a set of wrong treatment and lock system was needed to deal with sudden errors and realize safe share of resource. When instrument appeared $\mathrm{read} /$ write errors or read/write delay, it needed to notice control terminal timely, otherwise will cause blocked, which appear synchronization errors. Therefore, agent and control terminal needed re-synchronization. For different interface instrument, NA needed to select a unified interface, which to guarantee versatility and interchangeability. NA itself needs to support different interfaces instrumentations, but interface bus of current various instrumentations varied widely, so the compiled software for different instruments has very poor interchangeability and no versatility ${ }^{[6]}$. 
Therefore, NA was compiled based on virtual instrument software architecture, VISA should responsible for the unity of each interface.

Basic principle of VISA is to encapsulate different interfaces abstractly, which provides uniform interface for upper layer. Upper layer could be configured and programmed for interface of USB serial port GPIBVXI PXI and Ethernet through this interface, which achieve the unity of I/O interface software. VISA with bottom-up structural model also created a unified I/O control function set, which was VISA library. NA realized the feedback of instrument control measuring result through a call to these VISA library function.

\section{AODV Routing Algorithm in Mesh Network}

AODV routing algorithm is an on-demand routing. Its processing is faster and quick response to the change of link-state. AODV is the combination of DSR and DSDV, and has the advantage of both. Therefore, when realizing in Linux system, we choose AODV as WMN routing protocol. When realizing AODV protocol, if all the codes are compiled into the kernel of Linux system, it needs to compile the whole kernel, so it consumed more time to compile each; meanwhile, the stability of system cannot be guaranteed. Therefore, this approach is not appropriate. Thus, this paper divided AODV protocol into two parts: one part is kAODV module locating in the kernel of Linux; the other part is AODV daemon ${ }^{[7]}$ working in the user space. Kernel module is responsible for routing table search, dealing expiration and mistake, gateway solution, the packet, buffering no routing packet, and provide control interface of netlink routing table and Daemon Finger interface. Daemon Finger in user space is in charge of generating, receiving, handling of AODV frame and updating routing table. So it could reduce the size of the kernel and process low priority event in user space. However, it also has the defect that data communication in kernel space and user space causes additional expenses, process is delayed and total delayed time of routing operation is increased. The framework of AODV protocol implementation is shown as Fig. 2.

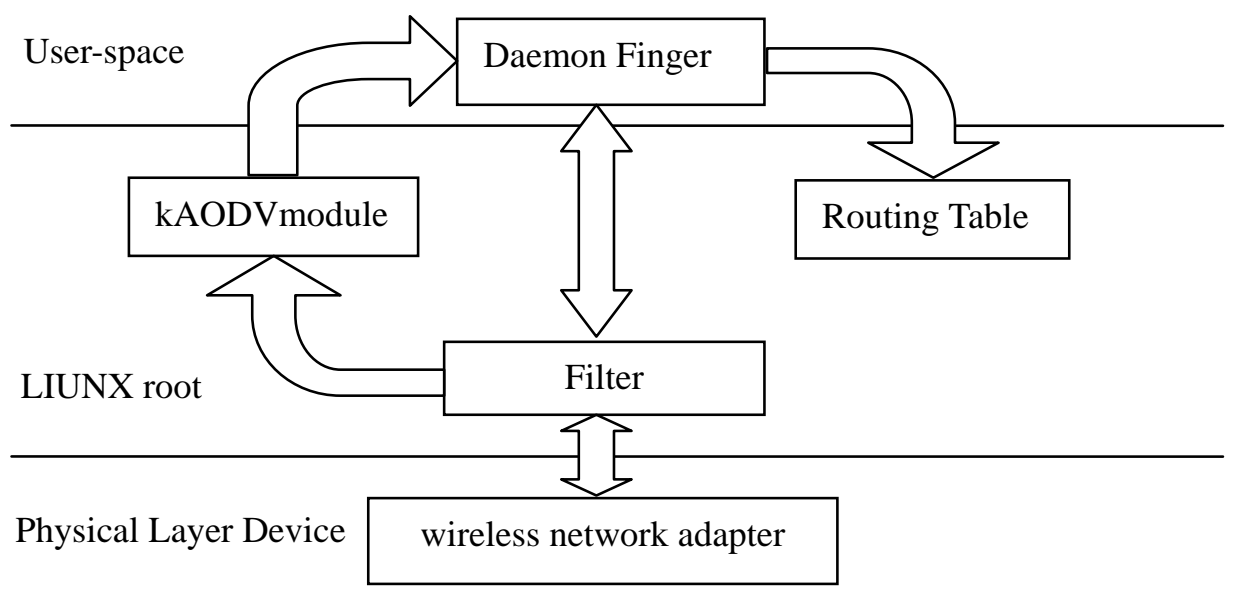

Fig. 2 Framework of Protocol Implementation

\section{NA Function Realization}

Agent and control terminal is linked with instrument through interconnection of HiSLIP, so VISA interface is invoked, and VISA calls the corresponding interface driver. Therefore, the driver requested by instrument is installed in the corresponding hardware environment in the early stage and realized HiSLIP protocol ${ }^{[8]}$. This paper used the oscilloscope with the Tektronix model of TDS2014B, its interface is USB, agent platform is S3C6410〈Linux, so USBTMC driver is needed to compile into development board ${ }^{[9]}$. 


\subsection{Network Communication Protocol}

IVI Alliance proposed High-speed LAN Instrument Protocol (HiSLIP) ${ }^{[10]}$ for remote instrument control. This protocol mainly included: separation instrument, local/remote state control of instrument, locking mechanism of instrument, service request, message end mechanism, interrupt mechanism of error detection. The structure of protocol itself is clear and the form is concise, which is easy for realization. HiSLIP header fix at $16 \mathrm{~B}$, which includes message parameter and data length of data package flag message type control code, the message format is shown as Fig. 3.

\begin{tabular}{|c|c|c|c|}
\hline $\begin{array}{c}\mathrm{H} \\
(16 \mathrm{~B})\end{array}$ & $\begin{array}{c}\mathrm{S} \\
(16 \mathrm{~B})\end{array}$ & $\begin{array}{c}\text { Type of Message } \\
\text { (1B) }\end{array}$ & $\begin{array}{l}\text { Control Code } \\
\text { (1B) }\end{array}$ \\
\hline \multicolumn{4}{|c|}{$\begin{array}{l}\text { Message Parameters } \\
\text { (4B) }\end{array}$} \\
\hline \multicolumn{4}{|c|}{$\begin{array}{l}\text { Data Length } \\
\text { (8B) }\end{array}$} \\
\hline \multicolumn{4}{|c|}{$\begin{array}{l}\text { Date } \\
(16 B+)\end{array}$} \\
\hline
\end{tabular}

Fig. 3 The format of HiSLIP message

The definition of each field in frame structure is as follow:

(1) "H", "S": the content fixes at 16B, which is used to quick test whether data packet has error.

(2) Message type: 1B, which is used to set the type of transmitted data package.

(3) Control code: $1 \mathrm{~B}$, which normally is relevant parameter of this message, which will change in terms of message type.

(4) Message parameter: 4B, it has multi-usage in data packet.

(5) Data length: 8B, which is used to identify the number of byte in data field.

(6) Data: the length of byte is not fixed, it depends on the length of data, which is used to load transmitted data message.

In terms of function, the message type roughly has 26 types: lock message, initialization message, trigger message, data message, status message, asynchronous channel message, control message and so on. When agent is initialized, it will create two TCP connections at the same interface (Suggested port number 4880 which assigned by IANA or appointed other port number), one is used as synchronizing channel, and the other is used as asynchronous channel. It could send bidirectional command in synchronizing channel, and agent packed the data according to the format of HiSLIP data package, then pass to lower TCP for encapsulation ${ }^{[11]}$. Both could send the message in specified type according to the format and sequence set by HiSLIP protocol, which is to achieve the information exchange between instrument and user. HiSLIP protocol is run in NA, which used as the server side of protocol. When the protocol starts up, both used suggested port number 4880 which assigned by IANA or appointed other port number. The process of implementation of Socket protocol by NA mainly includes protocol start-up, connection establishment, data exchange and fault-tolerant handling. The details are shown as Fig. 4. 


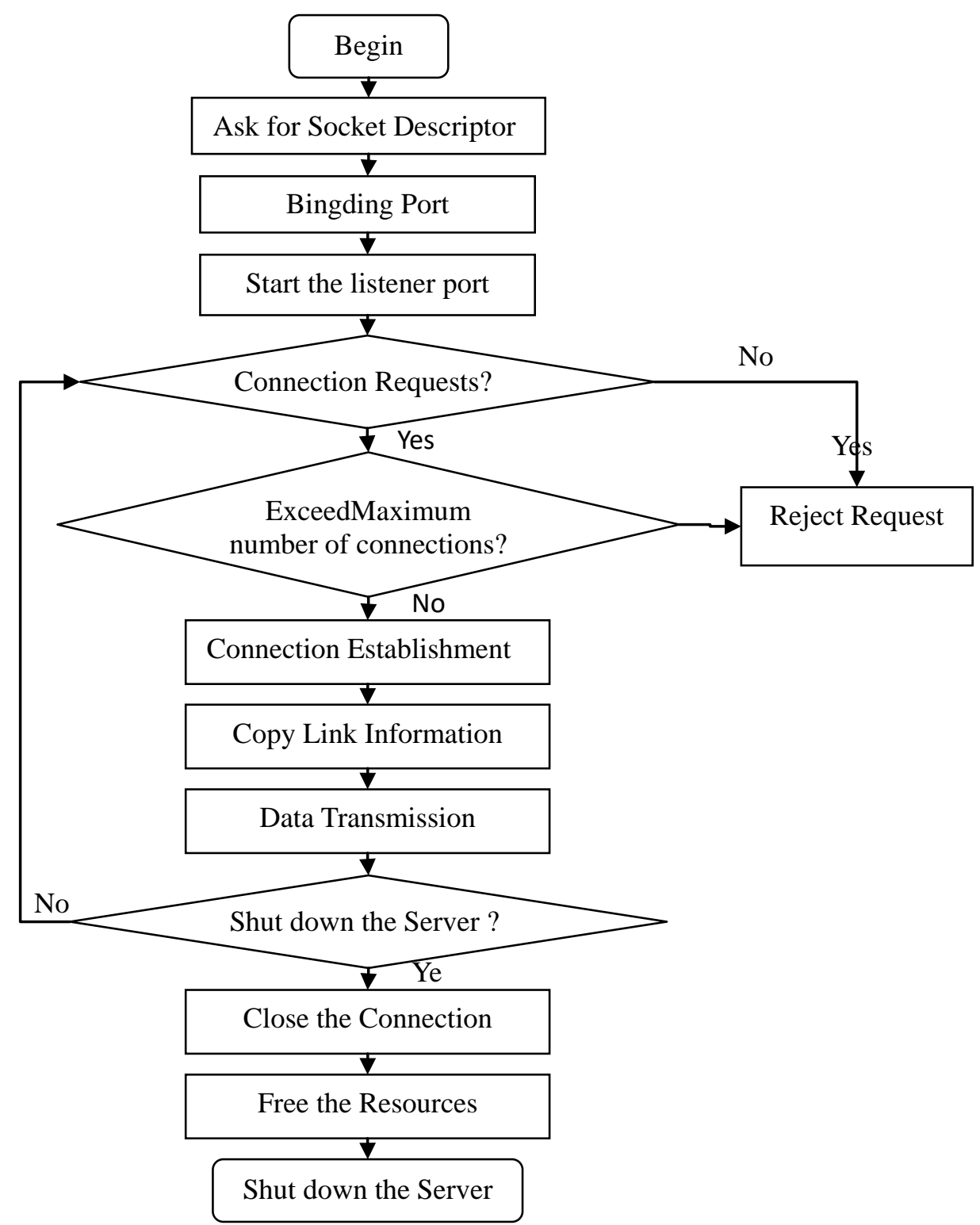

Fig. 4 Flowchart of establishing Socket link by Net Agent

\subsection{Test Result}

This paper realized NA function in embedded S3C6410\Linux platform. First, written agent program was transplanted to NA through cross compilation, and the corresponding interface driver and VISA were transplanted to agent platform; then visual soft panel in operation terminal computer called VISA library in the agent, and NA realized exact control of instrument and read of measured value. After NA obtained measured value or wave-shaped data, data will be fed back to soft panel timely. Experiment result showed that virtual instrument soft panel could show the wave shape of real instrument timely and correctly and various operation and the setting of instrument smoothly, such as the setting of trigger mode (coupled mode) and the detection of instrument.

\subsection{Summary}

This paper combined wireless Mesh network and instrument network agent for common interface instrumentation and proposed instrument NA design and Implementation based on WMN. Specific experiment indicated that the scheme had higher feasibility and better implementation 
effect. Due to easy usage and convenience to transplant, it is suitable for various plat form and easy to control cost, so it is easy for promotion. Overall, the transfer rate of data is lower due to protocol overhead and shared channel characteristics of wireless network (i.e. exposed terminal problem). Therefore, it still needs further improvement for future WMN routing protocol.

\section{Acknowledgements}

This work is supported by:

1) Key Laboratory of Nanchang City\& Special Majors in Jiangxi Province (NGTSZY201001)

2) Science and Technology Planning Project of the Department of Education (GJJ-15-12-39)

3) Science and Technology Planning Project of the Department of Education (GJJ-16-16-79)

4) The Research Project of Jiangxi Higher Education Reform (JXJG-16-3-19)

5) The Research Project of Teaching Reform of Degree and Postgraduate Education in Jiangxi (JXYJG-2012-037)

\section{References}

[1]http://baike.baidu.com/link?url=6HCU48BK1dkR5HEBrVpSQEDPg2v7TrNpkkX-rICj3dpFeghD9YP0evBKK RsRCercRFVtxdn4VT67Jcu2n2gX4K[EB/OL]

[2] Liu Jing, Li Youmou, Huang Jianjun, Zhou huan. Instrument Design and Implementation based on Wireless Mesh Network [J]. Modern Electronic Technology, 2017, 40(2):26-29.

[3] Luo Jin, Meng Chen.Research on Interchangeable Technology of Instrument [J]. Instrument and Meter Device, 2005(4): 26-28.

[4] Wu Ying, Qin Shuren, Zhang Fan. Design of Embedded Virtual Instrument based on USB bus [J]. Automatic Instrument, 2006, 25(3): 14-17.

[5] Zhang Yuping, Rong Jianhua, Fu Junqing. Research on Network Communication Technology based on Lab VIEW [J]. Automatic Instrument, 2004, 25(4): 21-23.

[6] An Youlin, Yang Suochang, Huang Kaoli, Research of Networked ATS Software Platform Based on Functional Interface [J].Computer Measurement\&Control,2007, 15(6):810-812

[7] Song Limei. Research and Implementation of Wireless Mesh Network Protocol based on WLAN [D]. Xi'an: Xi'an Electronic and Science University, 2008.

[8] Peng Jun, Zhu Yajia, Application of Multi-Agent System in Agricultural Information Intelligence Retrieves [J]. Micro-computer Information, 2008, 24(11):244-246

[9] Zhang Wen. Research and Implementation of LXI Multifunction Instrument System Software based on Embedded Linux Operating System [D]. Xi’an: Xi’an Electronic and Science University, 2008.

[10] IVI Foundation. IVI-6.1: high-speed LAN instrument protocol (HiSLIP) [EB/OL].http: //www.Ivifoundation.Org.

[11] Guo Meng, Li Youmei, Li Xiaofeng. Design and Realization of the Embedded Network Agent for Instrumentation [J]. Automatic Instrument, 2013, 34(12): 66-69. 